\title{
Metacarpal chondrosarcoma, from negligence to rareness : a case report and review of the literature
}

\author{
Pauline Ollero, Sophie Vanden Dungen, Katerina Cermak, Louis Kinnen
}

From the CHIREC Hospital, Braine-l'Alleud-Waterloo, Belgium

Chondrosarcoma is rarely found in the extremities but it remains the most common primary malignant bone tumor of the hand. We report an unusual case of a 46-year-old man with a huge chondrosarcoma on his left hand that has been evolving for more than 30 years. The mass has always been painless, the symptoms were only the deformation and a slight loss of motion. We did a subtotal resection since the patient refused the amputation. The investigation, which included thoracoabdominal tomography, scintigraphy and blood analysis, turned out to be negative. In the literature, CS are usually associated with a locally destructive growth but metastasis hasn't been often described. CS seems to be an aggressive tumor locally but, unlike in other sites, it seems to rarely metastasize when in the hands.

Keywords : chondrosarcoma ; hand ; tumor ; metastasis.

\section{INTRODUCTION}

Chondrosarcoma (CS) is the most common primary malignant bone tumor of the hand, although the incidence is low when compared to that of other sites $(1.5 \%)$. Independently of the location, CS is usually treated with surgical resection because it does not respond to chemotherapy or radiation. Although they may be primary tumors (1). CS are more likely coming from the malignant degeneration of a pre-existing lesion. CS of the hands are often described as a painful swelling that we usually

No benefits or funds were received in support of this study. None of the authors have a conflict of interest. remove promptly. As a consequence, we're not used to see long-lasting chondrosarcomas in the hands and aren't able to define the risk of metastases. The aim of this paper is to report an unusual case of a huge chondrosarcoma of the ulnar side of the hand that has been evolving for more than thirty years and to review the literature.

\section{CASE REPORT}

A 46-year-old male patient presents to the hand clinic for an expansile mass of the ulnar side of his left hand. He is a right-handed electrician. He reports he has a hospital phobia, the reason why he has waited to go see a surgeon until he couldn't wear a jacket anymore. He doesn't feel any pain. The range of motion of the fifth finger is slightly restricted, due to the volume of the tumefaction. (Figure 1) When he was 13, he went to see a general practitioner about a small swelling on his hand and has been told not to do anything if it doesn't cause pain. We don't know how it looked at this moment

Pauline Ollero M.D.

Sophie Vanden Dungen M.D.,

Katerina Cermak M.D.,

- Louis Kinnen M.D.

CHIREC Hospital, Braine-l'Alleud-Waterloo, Belgium

Correspondence : Pauline OLLERO, 19 rue champ du roi, 1040 Etterbeek, Belgium.

Email : pauline.ollero@hotmail.com

- 2021, Acta Orthopædica Belgica. 


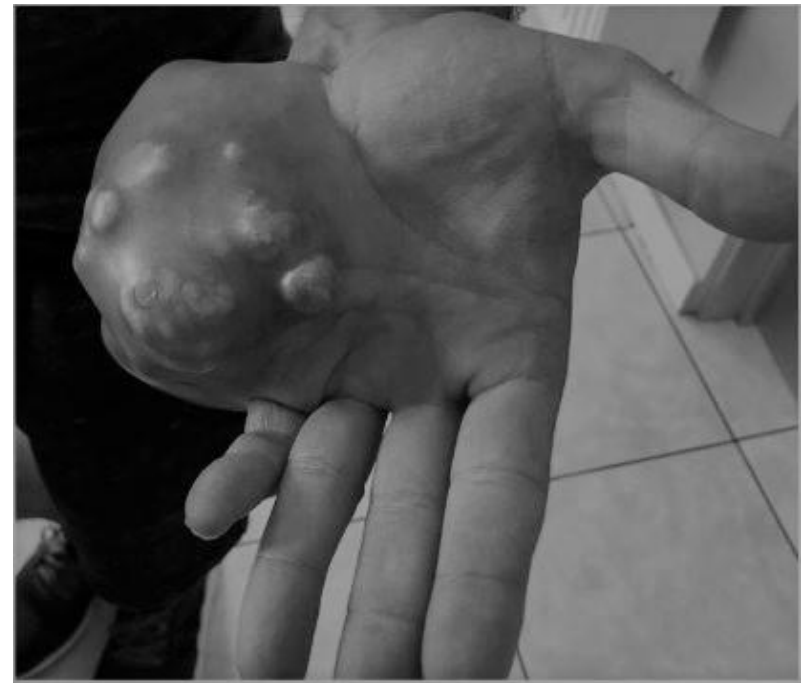

Figure 1. - Photograph showing the swelling of the left hand.

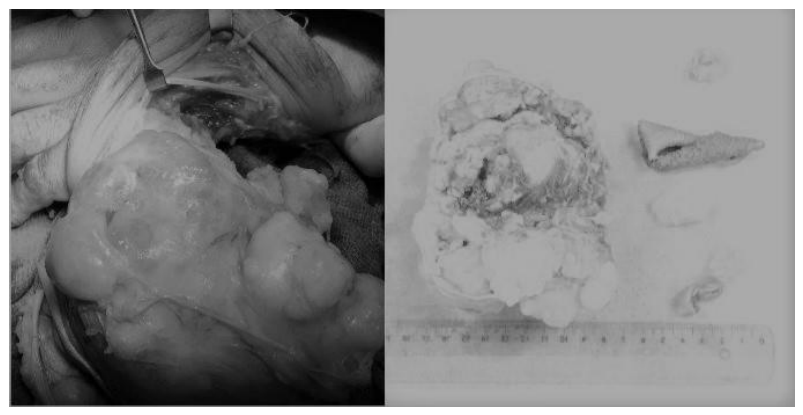

Figures 3. - Intra-operative photos of the tumor.

(enchondroma-like vs chondrosarcoma-like). Plain radiography (Figure 2) shows a massive bone tumor coming from the metacarpophalangeal joint with punctate calcifications, cortical destruction, bone expansion and a large soft-tissue extension. Thoracoabdominal tomography, scintigraphy and blood analysis were normal. An anterior approach has been used to remove the tumor (Figure 3). The nerve and extensor tendons were stuck in the tumor and had to be dissected. As the tumor was dissected, a fracture of the fifth metacarpal bone occurred. Since the patient was petrified about the surgery and refused any amputation that alters the normal appearance of his hand, we did not amputate and we fixed the bone with a plate (Figure 4). The tumor was diagnosed as a grade 2 chondrosarcoma. Although the resection of the CS didn't achieve

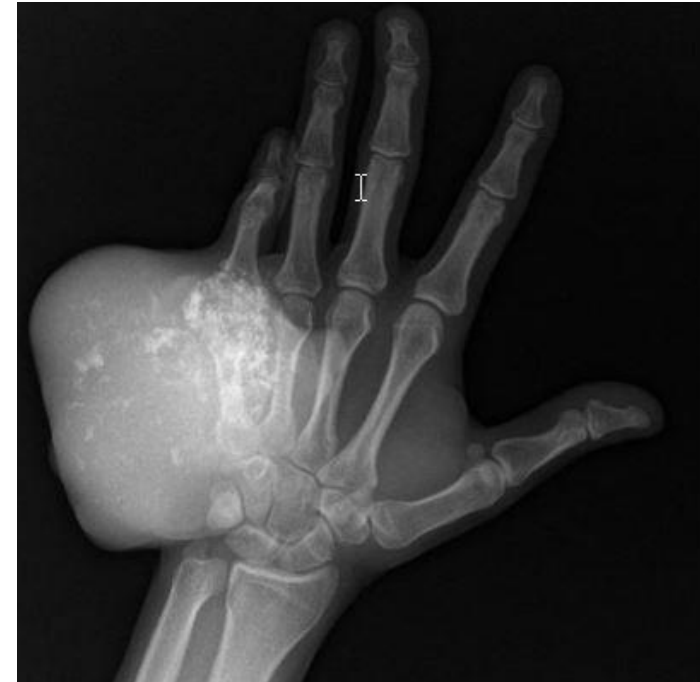

Figure 2. - Plain radiograph showing a lesion with punctate calcifications and an extension in the soft tissues.

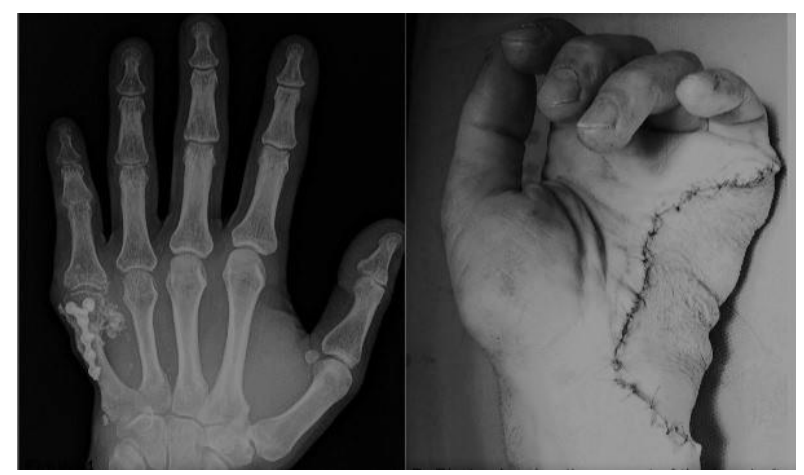

Figures 4. - Plain radiography of the left hand after the tumor resection showing a residual chondrosarcoma and a plate fixing the 5th metacarpal bone fracture. Photo showing the aspect of the hand after the resection.

wide margin and the amputation is required, the patient decided not to undergo another surgery.

\section{DISCUSSION}

$\mathrm{CS}$ is a malignant tumor with hyaline cartilage differentiation often found in the bones of pelvis and rarely in the hands (2). Bovée et al. also mentioned that CS of the hand is locally aggressive but, unlike CS elsewhere, rarely metastasizes. Indeed, they followed 38 cases of phalangeal CS for recurrence and metastasis. 10/15 tumors treated by local curettage recurred, $0 / 13$ treated with radical surgery recurred. Roberts and Price said that the usual clinical 
presentation is a progressively growing tumor, near the metacarpophalangeal joint (3). In their article, 19 $\mathrm{CS}$ are followed and no one metastasizes. Patil et al. studied $23 \mathrm{CS}$ of the hand, none had metastases whether they did excision or ray resection (4). 5 of $8 \mathrm{CS}$ treated with curettage recurred, none in the group "ray resection/amputation". Since the most common first symptom is tumefaction, we are not used to see long-lasting evolution as in the present case. Hatori et al. suggests that the median age of the patients at the time of diagnosis was over 60 , which also makes our case rare (5). CS can be primary or based upon a preexisting benign lesion like osteochondroma or enchondroma. Unfortunately in our case, there was no previous evaluation of this patient's lesion, so we don't know whether it was primary or secondary (6). The treatment required for high grade chondrosarcomas is radical resection or amputation (7).Unfortunately, the patient refused the optimal treatment. Thus, we will keep on following the evolution and hope no metastasis will occur.

\section{CONCLUSION}

Chondrosarcoma is not often found in the hands but, when it is, it acts like a locally aggressive tumor. It affects not only the bones but also the soft tissues. In most of the case reports, it is described as a painful swelling but our patient never felt any pain. So, we have to be aware, an indolent mass can also be malignant. It is well described that CS in the hands rarely metastasizes and, indeed, in the present case, the tumor has been evolving for many years and no metastasis has been found.

\section{REFERENCES}

1. García-Jiménez A, Chanes-Puiggrós C, Trullols-Tarragó L, Pulido-García M-C. Chondrosarcoma of the Hand Bones : A Report of 6 Cases and Review of the Literature. $J$ Hand Surg Asian-Pac Vol. 2019 ; 24(01) : 45-49.

2. Bovée JV, van der Heul RO, Taminiau AH, Hogendoorn PC. Chondrosarcoma of the phalanx : a locally aggressive lesion with minimal metastatic potential : a report of 35 cases and a review of the literature. Cancer Interdiscip Int $J$ Am Cancer Soc. 1999 ; 86(9) : 1724-1732.

3. Roberts PH, Price CH. Chondrosarcoma of the bones of the hand. J Bone Joint Surg Br. 1977 ; 59(2) : 213-221.

4. Patil S, De Silva MVC, Crossan J, Reid R. Chondrosarcoma of small bones of the hand. J Hand Surg. 2003 ; 28(6) : 602608.

5. Hatori M, Watanabe M, Kotake H, Kokubun S. Chondrosarcoma of the ring finger : a case report and review of the literature. Tohoku J Exp Med. 2006 ; 208(3) : 275-281.

6. Knapp P,Aviles A, Najarian C. Low-Grade Chondrosarcoma of the Proximal Phalanx : A Rare Presentation. Case Rep Orthop. $2019 ; 2019$.

7. Bashir SI, Gupta R, Khan HN, Ahmed R, Mohd A, Salaria AQ. Trans-articular chondrosarcoma grade 2 of proximal phalanx resulting in its fracture along with destruction of middle phalanx of 2 nd toe right foot : a case report and review of the literature. Cases J. 2009 ; 2(1) : 7488. 